\section{Usefulness of polyglycolic acid sheet for tumor hemorrhage from advanced gastric cancer}

\section{To the Editor,}

Gastrointestinal (GI) malignancies are responsible for $2-8 \%$ of upper GI bleedings [1]. Advanced gastric cancer accounts for $58 \%$ of these cases [2]. Surgical gastrectomy may be a reliable treatment, but most patients have a poor general condition. Embolization of the small-caliber vessel is difficult by interventional radiotherapy and sometimes induces tumor necrosis. Endoscopic methods use hemostatic forceps and argon plasma coagulation; however, adequate evidence is not available [3]. Recurrent bleeding within 30 days after successful endoscopic hemostasis occurs in up to $28 \%$ of patients due to tumor angiogenesis. Unsuccessful hemostasis leads to a poor prognosis and makes future therapy difficult. Thus, the development of a novel endoscopic procedure is required.

The polyglycolic acid (PGA) sheet (NEOVEIL ${ }^{\circledR}$ Gunze Ltd., Tokyo, Japan) reinforces and promotes wound healing. Several reports have described the effectiveness of PGA sheets in preventing recurrent bleeding and in managing delayed perforation after endoscopic submucosal dissection [4]. However, their effect in the management of advanced gastric cancer remains unknown. We herein describe the first use of PGA sheet for hemostasis and prevention of recurrent bleeding from advanced gastric cancer.

A 60-year old man with advanced gastric cancer and anticoagulant treatment for cardiovascular disease developed hematemesis and hemorrhagic shock. Contrast-enhanced computed tomography revealed arterial extravasation in the body of the stomach. Urgent upper GI endoscopy found spurting bleeding from the gastric tumor. Endoscopic procedure using hemostatic forceps was partially efficient, but the oozing hemorrhage continued. Consequently, a PGA sheet was introduced to achieve complete hemostasis and prevent recurrent bleeding. After the PGA sheet was fixed using hemostatic clips on both sides of the floor edge, fibrin glue was sprayed to fasten the sheet. Three days later, the PGA sheet completely covered the ulcerative floor of the cancer, preventing recurrent bleeding (Fig. 1). Seven days later, total gastrectomy was performed without recurrent bleeding or procedure-related complications.

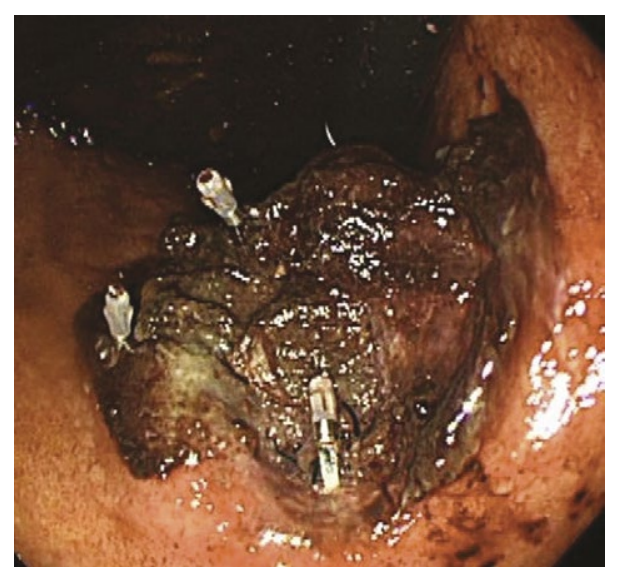

Fig. 1. Endoscopic image showing the PGA sheet covering the ulcer floor of the advanced gastric cancer.

In the present case, we report a new indication for the PGA sheet. However, PGA sheets still have unresolved issues: it is difficult to keep the sheet dry and retain its shape during the procedure. The optimal delivery and placement methods of PGA sheets have not been established. We delivered and placed the PGA sheet using wafer paper and thread as advocated by Kobayashi et al. [5]. Use of a PGA sheet for advanced gastric cancer complicated with bleeding has several advantages: avoidance of emergency surgery, continuation of chemotherapy, and maintenance of palliative care.

This case suggests that a PGA sheet can be an alternative option for managing tumor hemorrhage from advanced gastric cancer. 
Kazuhiro Kozuka, Hideki Kobara, Noriko Nishiyama, Hirohito Mori, Tsutomu Masaki

Department of Gastroenterology and Neurology, Faculty of Medicine, Kagawa University 1750-1 Ikenobe, Miki, Kita, Kagawa 761-0793, Japan

Correspondence: Kazuhiro Kozuka, koduka2525@gmail.com

Conflicts of interest: None.

DOI: $10.15403 /$ jgld-479

\section{REFERENCES}

1. Kawabata H, Hitomi M, Motoi S. Management of bleeding from unresectable gastric cancer. Biomedicines 2019;7:E54. doi:10.3390/ biomedicines 7030054

2. Kim YI, Choi IJ. Endoscopic management of tumor bleeding from inoperable gastric cancer. Clin Endosc 2015;48:121-127. doi:10.5946/ ce.2015.48.2.121

3. Song IJ, Kim HJ, Lee JA, et al. Clinical outcomes of endoscopic hemostasis for bleeding in patients with unresectable advanced gastric cancer. J Gastric Cancer 2017;17:374-383. doi:10.5230/jgc.2017.17.e42

4. Takimoto K, Hagiwara A. Filling and shielding for postoperative gastric perforations of endoscopic submucosal dissection using polyglycolic acid sheets and fibrin glue. Endosc Int Open 2016;4:E661-E664. doi:10.1055/s-0042-105867

5. Kobayashi N, Mori H, Kobara H, Masaki T. Reliable procedure of polyglycolic acid sheet delivery and placement on gastric artificial floor: application of wafer paper and thread. Dig Liver Dis 2018;50:724. doi:10.1016/j.dld.2018.01.129

\section{A misleading case of elevated alkaline phosphatase in alcoholic liver disease: Paget's disease of the bone}

\section{To the Editor,}

Alkaline phosphatase (AP) is a homodimeric protein enzyme present in all tissues throughout the body. Therefore, elevated levels of the enzyme are encountered in a large number of conditions including: hepatobiliary diseases (biliary obstruction, acute cholecystitis, hepatitis, liver cirrhosis, alcoholic liver disease) and extradigestive diseases, such as osteoblastic bone tumors, osteoporosis, myelofibrosis, leukemoid reaction, lymphoma, sarcoidosis, hyperthyroidism, hyperparathyroidism and myocardial infarction $[1,2]$. We report here the case of misleading elevated levels of AP in a 61 -year old male patient. This heavy drinker (80g alcohol/day for many years) presented in the emergency room for dizziness. Hepatomegaly with smooth edges was detected, without splenomegaly, abdominal tenderness or mass. Increased level of AP (225 IU/L) was detected and attributed to alcoholic liver disease. Apart from the AP, all other functional liver tests were normal. Abdominal ultrasound revealed liver steatosis grade I. Besides alcoholic liver disease, he was diagnosed with essential hypertension stage II, abdominal aortic atherosclerosis, hypercholesterolemia and benign prostatic hyperplasia. The elevation of AP was explained by the alcohol-related liver disease. The recommendations were to stop alcohol consumption and take phosphatdylcholine $1800 \mathrm{mg} /$ day.

At the follow up visit after three months, the AP was even higher: $603 \mathrm{IU} / \mathrm{L}$ although the patient had stopped alcohol consumption. He reported mild pain with decreased mobility in the left arm and shoulder with a sudden onset. Therefore, other potential extradigestive sources of high AP were investigated. The X-ray of the left arm for the pain showed a central osteolytic lesion with peripheric osteosclerosis of the left humerus which modified the bone shape, raising the suspicion of a bone tumor. Bone scintigraphy with Technetium 99-MDP revealed an increased signal in the left humerus. The bone biopsy showed irregular broad trabeculae, profound bone resorption with a high number of large osteoclasts with numerous nucleii, typical for Paget's disease of the bone (PDB).

Alendronic acid 70mg, 1 tablet/week, calcium supplements and vitamin $\mathrm{D}$ were initiated. At 3 and 6 months folow-up visits, a positive clinical outcome was noted with the disappearance of pain and normal mobility in the left arm and shoulder; the AP levels were normal.

Paget's disease of the bone or osteitis deformans is defined as a focal bone disorder caused by excessive osteoclastic activity, leading to bone resorption accompanied by an aberrant osteoblastic bone formation. The abnormalities occur in all phases of the remodelling process, resulting in highly fragile, augmented, deformed and more vascularized bones [3-5].

We emphasize the importance to be aware of the coexistence of a misleading extrahepatic disease in the presence of elevated AP in patients with alcoholic liver disease.

Ștefan L. Popa, George I. Golea, Liliana David

Iuliu Hațieganu University of Medicine and Pharmacy, Second Medical Department, Cluj-Napoca, Romania

Correspondence: Ștefan L. Popa, popa.stefan@umfcluj.ro

Conflicts of interest: None.

DOI: $10.15403 /$ jgld-613

\section{REFERENCES}

1. Millán JL, Whyte MP. Alkaline Phosphatase and Hypophosphatasia. Calcif Tissue Int 2016;98:398-416. doi:10.1007/s00223-015-0079-1

2. Mirhafez SR, Farimani AR, Dehhabe M, et al. Effect of Phytosomal Curcumin on Circulating Levels of Adiponectin and Leptin in Patients with Non-Alcoholic Fatty Liver Disease: A Randomized, DoubleBlind, Placebo-Controlled Clinical Trial. J Gastrointestin Liver Dis 2019;28:183-189. doi:10.15403/jgld-179

3. Shaker JL. Paget's disease of bone: A review of epidemiology, pathophysiology and management. Ther Adv Musculoskelet Dis 2009;1:107-125. doi:10.1177/1759720X09351779

4. Siris E. Indications for medical treatment of Paget's disease of bone. In: Singer FR, Wallach S. (eds.). Paget's Disease of Bone: Clinical Assessment, Present and Future Therapy. New York: Elsevier; 1991.

5. Thomas DW, Shepherd JP. Paget's disease of bone: Current concepts in pathogenesis and treatment. J Oral Pathol Med 1994;23:12-16. doi:10.1111/j.1600-0714.1994.tb00247.x 